(C2008 IEEE. Personal use of this material is permitted. However, permission to reprint/republish this material for advertising or promotional purposes or for creating new collective works for resale or redistribution to servers or lists, or to reuse any copyrighted component of this work in other works must be obtained from the IEEE 


\title{
Analysis of Optimum Frame Synchronization Based on Periodically Embedded Sync Words
}

\author{
Marco Chiani, Senior Member, IEEE, and Maria G. Martini, Senior Member, IEEE
}

\begin{abstract}
We present new tight bounds for evaluating the performance of sync word-based frame synchronization algorithms in the periodically embedded case. We consider antipodal signaling with coherent detection over additive white Gaussian noise and both optimal and suboptimal search techniques. Our bounds are very close to results obtained through simulation and tend asymptotically (for increasing signal-to-noise ratios) to the exact performance.
\end{abstract}

Index Terms-Frame synchronization, Massey's metric, sync words, synchronization probability, union bound.

\section{INTRODUCTION}

$\mathbf{O}$ $\mathrm{NE}$ of the key issues in digital communication systems is frame synchronization where, often, synchronization words are inserted in the bitstream to delimit frames [1]-[5].

A first intuitive approach to achieve frame synchronization could be to correlate the received signal with the expected sync word (SW) over a time window that contains exactly one inserted SW, looking for the position where this correlation output is maximum. This approach is possible only for the periodically embedded case, where sync words are equally spaced or, in other words, for constant known frame lengths. In the binary symmetric channel, this detection through correlation is optimal, whereas in the additive white Gaussian noise (AWGN) channel, this is not true: in fact, in [6], the optimal metric has been identified for the considered case of fixed-length frames, and its approximations for high and low signal-to-noise ratio (SNR) have been provided. Although not optimal, frame detection through correlation has become a common engineering practice. In [7], the performance evaluation through simulation of these metrics has been presented, whereas the analytical approach is considered too complicated for the metrics in [6] even for an approximate analysis. In [8], an extension of [6] is given and, for general $M$-ary phase-coherent and phase-noncoherent signaling, a union lower bound on synchronization probability for the correlation rule on AWGN channels is determined. Also, in [8], an analytical approach was considered too difficult, and, hence, the performance investigation for the optimal metric was carried out through simulation. Extensions and further analysis are provided in [9]-[12]. Synchronization for unknown frame lengths, where

Paper approved by E. Serpedin, the Editor for synchronization and Sensor Network of the IEEE Communications Society. Manuscript received February 2006; revised November 2006. This work was supported by the European Commission under Project FP6 IST-001812 "PHOENIX.” This paper was presented in part at the IEEE Vehicular Technology Conference, Stockholm, May 2005.

M. Chiani is with WiLAB, University of Bologna, 40136 Bologna, Italy (email:marco.chiani@unibo.it).

M. G. Martini was with CNIT/DEIS, University of Bologna, 40136 Bologna, Italy. She is now with Kingston University, London, UK (e-mail: mgmartini@ ieee.org).

Digital Object Identifier 10.1109/TCOMM.2007.908510

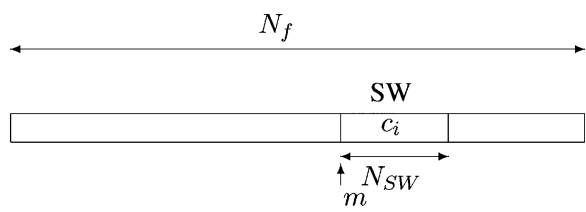

b)

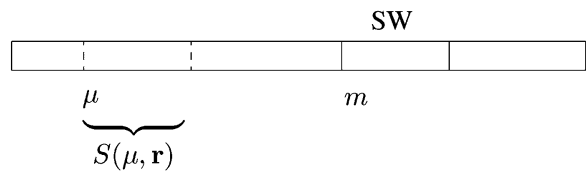

c)

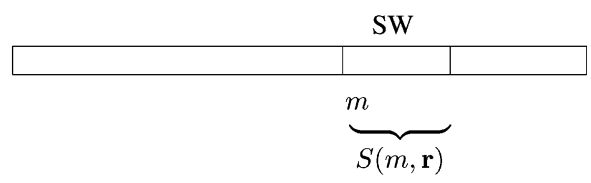

d)

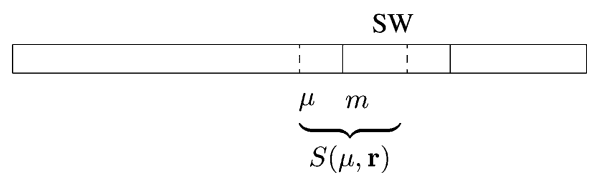

Fig. 1. (a) Frame structure. (b) Case $|\mu-m| \geq N_{\mathrm{SW}}$ (pure data case). (c) Case $\mu=m$. (d) Case $0<|\mu-m|<N_{\mathrm{SW}}$ ("mixed data/sync word" case).

the synchronization is based on a comparison of a proper metric with a threshold, is derived in [13] and [14] according to the hypothesis testing theory, and an exact analytical performance evaluation is provided.

In this paper we refer to frame synchronization for coherent detection and known constant-length frames, providing analytical lower bounds for the optimal and suboptimal metrics, thus solving a problem that was considered not analytically tractable since its derivation.

The remainder of this paper is organized as follows. In Section II, the problem of frame synchronization with periodically embedded sync words (SWs) is described. In Section III, the statistical distribution of the optimal metric and of its approximation is derived. In Section IV, a tight bound for the false synchronization probability is derived. Finally, numerical results are presented in Section V.

\section{On Locating Periodically EMBedded SynC WordS}

We consider the situation of Fig. 1(a) where a sync word composed of $N_{\mathrm{SW}}$ binary symbols $\left(c_{0}, \ldots, c_{N_{\mathrm{Sw}}-1}\right)$ is periodically inserted, with period $N_{f}$, in a random binary data stream. Each sync word symbol $c_{i}$ is either -1 or +1 . The data streams are assumed to be composed of symbols $d_{i} \in\{-1,+1\}$ that are independent, identically distributed (i.i.d.) random variables 
(r.v.s) with equiprobable -1 and +1 , and the bit intervals are assumed to be known at the receiver.

Let $^{1} \mathbf{d}=\left(d_{N_{\mathrm{SW}}}, d_{N_{\mathrm{SW}}+1}, \ldots, d_{N_{f}-1}\right)$ denote $N_{f}-N_{\mathrm{SW}}$ random data symbols and consider the concatenation $\mathbf{c d}=$ $\left(c_{0}, \ldots, c_{N_{\mathrm{SW}}-1}, d_{N_{\mathrm{SW}}}, \ldots, d_{N_{f}-1}\right)$. If the sync word actually begins in position $m$ of the received segment, we can express the received vector $\mathbf{r}=\left(r_{0}, \ldots, r_{N_{f}-1}\right)$ as

$$
\mathbf{r}=T^{m}(\mathbf{c d})+\mathbf{n}
$$

where $T(\cdot)$ is the cyclic shift operator defined as $T(\mathbf{c d})=$ $\left(d_{N_{f}-1}, c_{0}, \ldots, c_{N_{\mathrm{SW}}-1}, d_{N_{\mathrm{SW}}}, \ldots, d_{N_{f}-2}\right)$ and $\mathbf{n}$ is the noise vector whose elements $n_{i}$ are i.i.d. real Gaussian r.v.s each with zero mean and variance $\sigma^{2}$, so that the SNR is $E_{s} / N_{0}=$ $1 /\left(2 \sigma^{2}\right)$.

The optimum decision rule, in the sense of maximizing the probability of correctly locating the sync word, is to choose the estimate of the position $m$ as the value $\mu, 0 \leq \mu<N_{f}$, which maximizes the probability $\operatorname{Pr}\{m=\mu \mid \mathbf{r}\}$. Under the hypothesis that the parameter to be estimated $m$ is a priori equally likely to occur in any position, the aforementioned maximization is equivalent to determine the value $\mu$ that maximizes the metric given next [6].

Optimum Rule: Choose $\mu$ maximizing

$$
S_{\mathrm{O}}(\mu, \mathbf{r})=\sum_{i=0}^{N_{\mathrm{SW}}-1} c_{i} r_{i+\mu}-\sigma^{2} \sum_{i=0}^{N_{\mathrm{SW}}-1} \ln \cosh \frac{r_{i+\mu}}{\sigma^{2}}
$$

where the additions in subscripts like those in (2) are taken through the paper modulo $N_{f}$ to account for all possible positions of the sync word.

Optimum Rule-Approximation: Choose $\mu$ maximizing

$$
S_{\mathrm{H}}(\mu, \mathbf{r})=\sum_{i=0}^{N_{\mathrm{SW}}-1} c_{i} r_{i+\mu}-\sum_{i=0}^{N_{\mathrm{SW}}-1}\left|r_{i+\mu}\right| .
$$

Finally, neglecting the second term in (2), we obtain the correlation rule, still representing a common engineering practice, regardless of its suboptimality.

Correlation Rule: Choose $\mu$ maximizing

$$
S_{\mathrm{C}}(\mu, \mathbf{r})=\sum_{i=0}^{N_{\mathrm{SW}}-1} c_{i} r_{i+\mu}
$$

The performance of the approximation $S_{\mathrm{H}}(\mu, \mathbf{r})$ is generally very close to that of the optimal metric $S_{\mathrm{O}}(\mu, \mathbf{r})$, as shown through simulation in [7] and [8] and by our analytical results (see Section V). Given its higher implementation simplicity, it is, thus, a candidate to be used in most practical situations.

\section{STATISTICAL DiSTRIBUTION OF THE SYNCHRONIZATION METRICS}

For the received vector $\mathbf{r}$ defined in (1), let $S(\mu, \mathbf{r})$ denote the generic adopted metric as defined by (2), (3) or (4). In the synchronization process, we make an error if there exists at least

\footnotetext{
${ }^{1}$ In the following, we use, when possible, capital letters to indicate r.v.s and bold for vectors.
}

a position $\mu \neq m$ such that

$$
S(m, \mathbf{r})<S(\mu, \mathbf{r})
$$

where $m$ is the actual starting position of the sync word. ${ }^{2}$

Let us now indicate with $\mathbf{R}=\left(R_{0}, \ldots, R_{N_{f}-1}\right)$ the random vector corresponding to the vector $\mathbf{r}$. The probability of correct synchronization $P_{\mathrm{S}}$, is therefore

$$
P_{\mathrm{S}}=1-\operatorname{Pr}\left\{\bigcup_{\mu \neq m}[S(m, \mathbf{R})<S(\mu, \mathbf{R})]\right\} .
$$

Since, in general, the events in previous equation are not disjoint, we resort to the union bound. With this approach, the probability of correct synchronization is lower bounded as

$$
P_{\mathrm{S}} \geq P_{\mathrm{SL}} \triangleq 1-\sum_{\mu=0, \mu \neq m}^{N_{f}-1} \operatorname{Pr}\{S(m, \mathbf{R})<S(\mu, \mathbf{R})\}
$$

and the synchronization error probability, or false synchronization probability $P_{\mathrm{FS}}$, is upper bounded as

$$
P_{\mathrm{FS}}=1-P_{\mathrm{S}} \leq P_{\mathrm{FSU}} \triangleq 1-P_{\mathrm{SL}} .
$$

Note that for high SNR, errors are likely to occur due to only one position where (5) is verified. In other words, the events leading to the union bound tend to become disjoint for high SNR and the bound converges to the true $P_{\mathrm{S}}$, i.e., it is asymptotically tight.

The problem now is to evaluate the pairwise synchronization error probability (PSEP)

$$
\begin{aligned}
P_{\text {sep }} & \triangleq \operatorname{Pr}\{S(m, \mathbf{R})<S(\mu, \mathbf{R})\} \\
& =\operatorname{Pr}\{S(m, \mathbf{R})-S(\mu, \mathbf{R})<0\}, \quad \mu \neq m
\end{aligned}
$$

For the determination of (7), it is necessary to understand how the r.v.s $S(\mu, \mathbf{R})$ and $S(m, \mathbf{R})$ are distributed and their statistical interdependency. Here, since the metric $S(.,$.$) is evaluated$ over $N_{\text {SW }}$ consecutive received samples, we have three possible situations [cases (b)-(d) in Fig. 1]. The first is when the metric evaluation window is over samples due only to data symbols, which occur for $S(\mu, \mathbf{r})$ with $|\mu-m| \geq N_{\mathrm{SW}}$. The second is for $S(m, \mathbf{r})$ where the metric evaluation window is exactly coincident with the sync word. The third, which we call the "mixed data/sync word" case, is when the metric is calculated over some samples due to data and some due to the sync word. The latter is verified for $0<|\mu-m|<N_{\text {SW }}$. Due to the presence of AWGN, the r.v.s $S(\mu, \mathbf{R})$ with $|\mu-m| \geq N_{\mathrm{SW}}$ [pure data samples, Fig. 1(b)] and $S(m, \mathbf{R})$ [Fig. 1(c)] are statistically independent. So, we can easily study here the statistical distribution of $S(\mu, \mathbf{R})-S(m, \mathbf{R})$ starting from the individual distribution of $S(\mu, \mathbf{R})$ and of $S(m, \mathbf{R})$.

A different situation arises for the "mixed data/sync word" case [see Fig. 1(d)], where $S(\mu, \mathbf{R})$ and $S(m, \mathbf{R})$ have some samples in common. The analysis of this situation is carried out in [15] and not reported here for brevity. However, for properly

\footnotetext{
${ }^{2}$ When the same maximum value of the metric is found in more positions, we assume the synchronizer picks one randomly. Thus, an error can also occur when there is an equality in (5): this case is not considered for the moment, but will be discussed later.
} 
designed synchronization sequences and for large $N_{f} / N_{\mathrm{SW}}$ ratios, the "mixed data/sync word" case can be approximated by the pure data case of Fig. 1(b) [15].

With the aim of determining bounds on the synchronization probability, we now study the distribution of the metrics (2)-(4), in terms of the characteristic function (ch.f.), by using the results from [14].

\section{A. Characteristic Function of $S(\mu, \mathbf{R})$ When $|\mu-m| \geq N_{S W}$}

1) Optimum Rule: The distribution of the metric for the optimum rule can be more easily derived by observing that, apart from a scaling factor and a constant additional term that do not affect the test, the metric in (2) can be equivalently rewritten as

$$
S_{\mathrm{O}}(\mu, \mathbf{r})=-\sum_{i=0}^{N_{\mathrm{SW}}-1} \ln \left(1+e^{-\frac{2 r_{i+\mu} c_{i}}{\sigma^{2}}}\right) .
$$

Following the same reasoning as in [14] it is possible to show that the ch.f. of the i.i.d. r.v.s $Z_{i}=\ln \left(1+e^{-\left(2 R_{i+\mu} c_{i}\right) / \sigma^{2}}\right)$ is given by

$$
\begin{aligned}
\Phi_{Z}(\nu) \triangleq & \mathbb{E}\left\{e^{j 2 \pi \nu Z_{i}}\right\}=\frac{1}{\sqrt{2 \pi \sigma^{2}}} \\
& \times \int_{-\infty}^{\infty} e^{-\frac{r^{2}+1}{2 \sigma^{2}}}\left(1+e^{-\frac{2 r}{\sigma^{2}}}\right)^{j 2 \pi \nu} \cosh \left(\frac{r}{\sigma^{2}}\right) d r
\end{aligned}
$$

where $j=\sqrt{-1}$ is the imaginary unit. Then, the ch.f. of $S_{\mathrm{O}}(\mu, \mathbf{R})=-\sum_{i=0}^{N_{\mathrm{sw}}-1} Z_{i}$ is easily obtained from that of $Z_{i}$ as

$$
\Phi_{S_{\mathrm{O}}(\mu, \mathbf{R})}(\nu) \triangleq \mathbb{E}\left\{e^{j 2 \pi \nu S_{\mathrm{O}}(\mu, \mathbf{R})}\right\}=\left[\Phi_{Z}(-\nu)\right]^{N_{\mathrm{SW}}} .
$$

2) Optimum Rule-Approximation: In order to evaluate the statistical distribution of the metric in (3), by using the property $\left|c_{i}\right|=1$ we write

$$
S_{\mathrm{H}}(\mu, \mathbf{R})=\sum_{i=0}^{N_{\mathrm{SW}}-1} c_{i} R_{i+\mu}-\sum_{i=0}^{N_{\mathrm{SW}}-1}\left|c_{i} R_{i+\mu}\right| .
$$

In [13] and [14], it was shown that the ch.f. of r.v.s like $V_{i} \triangleq$ $c_{i} R_{i+\mu}-\left|c_{i} R_{i+\mu}\right|$ is

$$
\begin{aligned}
\Phi_{V_{i}}(\nu)= & \frac{1}{4} e^{4 \pi \nu\left(j-2 \pi \nu \sigma^{2}\right)}\left[\operatorname{erfc}\left(\frac{1+j 4 \pi \nu \sigma^{2}}{\sqrt{2} \sigma}\right)\right. \\
& \left.+e^{-j 8 \pi \nu} \operatorname{erfc}\left(\frac{-1+j 4 \pi \nu \sigma^{2}}{\sqrt{2} \sigma}\right)\right]+\frac{1}{2} .
\end{aligned}
$$

Then, since $S_{\mathrm{H}}(\mu, \mathbf{R})=\sum_{i=0}^{N_{\text {sw }}-1} V_{i}$, the ch.f. of the metric in (10) is given by

$$
\Phi_{S_{\mathrm{H}}(\mu, \mathbf{R})}(\nu) \triangleq \mathbb{E}\left\{e^{j 2 \pi \nu S_{\mathrm{H}}(\mu, \mathbf{R})}\right\}=\left[\Phi_{V}(\nu)\right]^{N_{\mathrm{SW}}},
$$

with $\Phi_{V}(\nu) \triangleq \Phi_{V_{i}}(\nu)$.

3) Correlation Rule: For completeness, we also report the analysis of the correlation metric (4), which has been studied by a different approach also in [8]. With our methodology, using the fact that conditionally to $d_{i+\mu}$, the r.v.s $\Psi_{i}=R_{i+\mu} c_{i}$ are i.i.d. Gaussian with same variance and mean $d_{i+\mu} c_{i}$, and the assumption $\operatorname{Pr}\left\{d_{i}=1\right\}=\operatorname{Pr}\left\{d_{i}=-1\right\}=1 / 2$, the ch.f. of $\Psi_{i}$ is easily obtained as

$$
\begin{aligned}
\Phi_{\Psi_{i}}(\nu) & \triangleq \mathbb{E}\left\{e^{j 2 \pi \nu \Psi_{i}}\right\} \\
& =\frac{1}{2} e^{-2 \pi \nu\left(-j+\pi \sigma^{2} \nu\right)}+\frac{1}{2} e^{-2 \pi \nu\left(j+\pi \sigma^{2} \nu\right)} \\
& =\cos (2 \pi \nu) e^{-2 \pi^{2} \nu^{2} \sigma^{2}}
\end{aligned}
$$

and, therefore,

$$
\Phi_{S_{\mathrm{C}}(\mu, \mathbf{R})}(\nu) \triangleq \mathbb{E}\left\{e^{j 2 \pi \nu S_{\mathrm{C}}(\mu, \mathbf{R})}\right\}=\left[\cos (2 \pi \nu) e^{-2 \pi^{2} \nu^{2} \sigma^{2}}\right]^{N_{\mathrm{SW}}} .
$$

\section{B. Characteristic Function of $S(m, \mathbf{R})$}

1) Optimum Rule: In this case the metric is (8) with $\mu=m$. Again, following [14], it is possible to show that the ch.f. of the independent r.v.s $X_{i}=\ln \left(1+e^{-\left(2 R_{i+m} c_{i}\right) / \sigma^{2}}\right)$ is

$$
\begin{aligned}
\Phi_{X}(\nu) & \triangleq \mathbb{E}\left\{e^{j 2 \pi \nu X_{i}}\right\} \\
& =\frac{1}{\sqrt{2 \pi \sigma^{2}}} \int_{-\infty}^{\infty} e^{-\frac{(r-1)^{2}}{2 \sigma^{2}}}\left(1+e^{-\frac{2 r}{\sigma^{2}}}\right)^{j 2 \pi \nu} d r .
\end{aligned}
$$

Then, the ch.f. of $S_{\mathrm{O}}(m, \mathbf{R})=-\sum_{i=0}^{N_{\mathrm{sw}}-1} X_{i}$ is obtained from that of $X_{i}$ as

$$
\Phi_{S_{\mathrm{O}}(m, \mathbf{R})}(\nu) \triangleq \mathbb{E}\left\{e^{j 2 \pi \nu S_{\mathrm{O}}(m, \mathbf{R})}\right\}=\left[\Phi_{X}(-\nu)\right]^{N_{\mathrm{SW}}} .
$$

2) Optimum Rule-Approximation: For the r.v. defined in (10), when $\mu=m$ we have that the r.v.s $R_{i}$ are Gaussian i.i.d.s with mean values $c_{i}$ and variance $\sigma^{2}$. Hence, $c_{i} R_{i+m}$ are still Gaussian with mean value $c_{i}^{2}=1$. In these conditions, following the approach in [13], the ch.f. of $Y_{i}=c_{i} R_{i+m}-\left|c_{i} R_{i+m}\right|$ can be shown to be

$$
\begin{aligned}
\Phi_{Y_{i}}(\nu)= & \frac{1}{2} \operatorname{erfc}\left(-\frac{1}{\sqrt{2} \sigma}\right) \\
& +\frac{1}{2} e^{4 \pi \nu\left(j-2 \pi \nu \sigma^{2}\right)} \operatorname{erfc}\left(\frac{1+j 4 \pi \nu \sigma^{2}}{\sqrt{2} \sigma}\right) .
\end{aligned}
$$

Then, we have the ch.f. of the metric in (10) for $\mu=m$ given by

$$
\Phi_{S_{\mathrm{H}}(m, \mathbf{R})}(\nu) \triangleq \mathbb{E}\left\{e^{j 2 \pi \nu S_{\mathrm{H}}(m, \mathbf{R})}\right\}=\left[\Phi_{Y}(\nu)\right]^{N_{\mathrm{SW}}}
$$

with $\Phi_{Y}(\nu) \triangleq \Phi_{Y_{i}}(\nu)$.

3) Correlation Rule: For the correlation metric, since the r.v.s $\Psi_{i}=R_{i+m} c_{i}$ are i.i.d. Gaussian with unitary mean value, the ch.f. is

$$
\Phi_{S_{\mathrm{C}}(m, \mathbf{R})}(\nu)=e^{-2 \pi \nu\left(-j+\pi \sigma^{2} \nu\right) N_{\mathrm{Sw}}} .
$$

\section{Bounds on the FAlSe SynChronization PRobability}

In this section, the PSEP is first evaluated and results are then used to evaluate the bounds on false synchronization probability. To obtain the PSEP, we study the distribution of $S(m, \mathbf{R})-$ $S(\mu, \mathbf{R})$ when $|\mu-m| \geq N_{\mathrm{SW}}$. This is the situation where the two metrics are evaluated as per the conditions of Fig. 1, cases (b) and (c). As previously observed, due to the AWGN, the r.v.s 
$S(m, \mathbf{R})$ and $S(\mu, \mathbf{R})$ are here statistically independent. Hence, the ch.f. of $\Xi=S(m, \mathbf{R})-S(\mu, \mathbf{R})$ is given by

$$
\Phi_{\Xi}(\nu) \triangleq \mathbb{E}\left\{e^{j 2 \pi \nu \Xi}\right\}=\Phi_{S(m, \mathbf{R})}(\nu) \Phi_{S(\mu, \mathbf{R})}(-\nu),
$$

where $\Phi_{S(m, \mathbf{R})}(\nu)$ and $\Phi_{S(\mu, \mathbf{R})}(-\nu)$ are given in the previous section. The PSEP for the pure data case $P_{\text {sep }}^{D}$, is therefore obtained from the cumulative distribution function (c.d.f.) of $\Xi, F_{\Xi}(\xi)$, as

$$
\begin{aligned}
P_{\text {sep }}^{D} & \triangleq \operatorname{Pr}\left\{S(m, \mathbf{R})<S(\mu, \mathbf{R}) \mid\left(|\mu-m| \geq N_{\mathrm{SW}}\right)\right\} \\
& =F_{\Xi}(0)=\frac{1}{2}-\int_{-\infty}^{\infty} \frac{\Phi_{\Xi}(\nu)}{j 2 \pi \nu} d \nu
\end{aligned}
$$

which can be easily numerically evaluated in no more than few seconds with standard mathematical packages.

Note that, when the c.d.f. of $\Xi$ has a discontinuity in 0 , meaning a nonzero probability of having $S(m, \mathbf{R})=$ $S(\mu, \mathbf{R})$, the inverse Fourier transform in (18) correctly returns $\left[F_{\Xi}\left(0^{+}\right)+F_{\Xi}\left(0^{-}\right)\right] / 2$, which corresponds to randomly choosing between the two positions in case of equality of the metrics. So, (18) implicitly includes the case when there are more maxima in the observation window.

To derive a bound on the false synchronization probability, we observe that the PSEP for the pure data case, expressed by (18), does not depend on $\mu$ provided that $|\mu-m| \geq N_{\text {SW }}$. Thus, we can write the union bound on the false synchronization probability as

$$
\begin{aligned}
P_{\mathrm{FSU}}= & \left(N_{f}-2 N_{\mathrm{SW}}+1\right) P_{\mathrm{sep}}^{D}+2 \sum_{J=1}^{N_{\mathrm{Sw}}-1} \operatorname{Pr}\{S(m, \mathbf{R}) \\
& -S(\mu, \mathbf{R})<0 \mid(|\mu-m|=J)\} .
\end{aligned}
$$

A simpler expression is obtained by approximating the "mixed data/sync word" case with the pure data case, leading to

$$
P_{\mathrm{FSU}} \simeq\left(N_{f}-1\right) P_{\text {sep }}^{D} \text {. }
$$

This expression requires only (18) and is very close to (19) for properly designed sequences [15].

\section{A. Asymptotic Performance}

The asymptotic value of the PSEP in (18) for increasing SNRs results in

$$
P_{\mathrm{ep} \infty}^{D}=\frac{1}{2^{N_{\mathrm{SW}}+1}} .
$$

This is due to the fact that for the noiseless channel, the probability of exactly emulating a sync word is $1 /\left(2^{N_{\mathrm{SW}}}\right)$ and, in case of emulation, there is a probability $1 / 2$ of randomly choosing the emulated sync word instead of the one inserted on purpose.

We now define the asymptotic union bound (AUB) as the bound in (20), obtained in the noiseless case. By substituting (21) in (20), we obtain

$$
\text { AUB }=\left(N_{f}-1\right) \frac{1}{2^{N_{\mathrm{SW}}+1}} .
$$

In the case of absence of noise, the probability of false synchronization in the "ramdom data limit" (RDL) case may also be evaluated. This limit has been derived in [7, eq. (13)] for

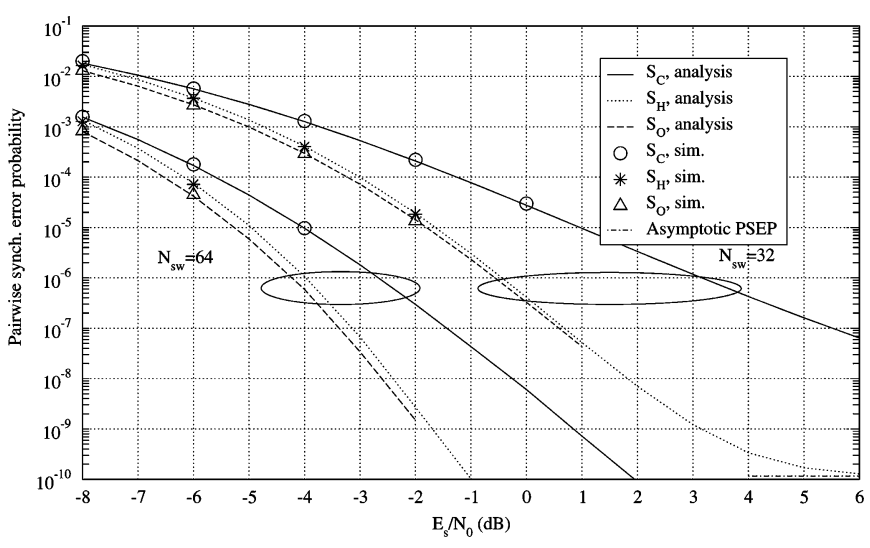

Fig. 2. Pairwise synchronization error probability versus $E_{s} / N_{0}=1 / 2 \sigma^{2}$. Sync word of length $N_{\mathrm{SW}}=32$ and $N_{\mathrm{SW}}=64$.

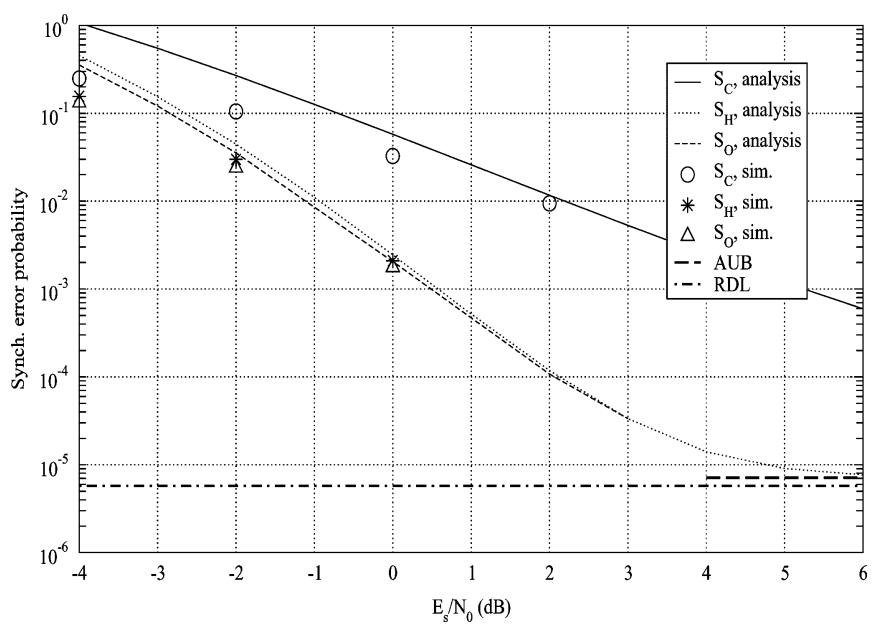

Fig. 3. False synchronization probability versus $E_{s} / N_{0}=1 / 2 \sigma^{2}$. Sync word of length $N_{\mathrm{SW}}=24$, frame length $N_{f}=240$.

frame synchronization sequences with distinct prefixes and suffixes property. It represents the ultimate lower bound for the probability of false synchronization: the fact that a pattern coincident with the frame synchronization sequence can occur in certain subsequences of the random data sequence ensemble implies that false synchronization on data can occur even when the channel is noiseless.

\section{NUMERICAL RESULTS}

We describe in this section some results obtained by using the previously presented analysis.

First, in Fig. 2 the PSEP (18) is evaluated for $S_{\mathrm{O}}$ and $S_{\mathrm{H}}$ for sync words of length $N_{\mathrm{SW}}=32$ and $N_{\mathrm{SW}}=64$, and validated by simulation results. The asymptotic PSEP $P_{\mathrm{ep} \infty}^{D}$ is also reported. These figures allow an easy derivation of the probability of false synchronization for any frame length $N_{f}$, according to (20). We may observe that the PSEP obtained considering the metric $S_{\mathrm{H}}$ and the one obtained with the metric $S_{\mathrm{O}}$ are very similar.

Examples of derivation of the probability of false synchronization are reported in the following figures. The case of sync 


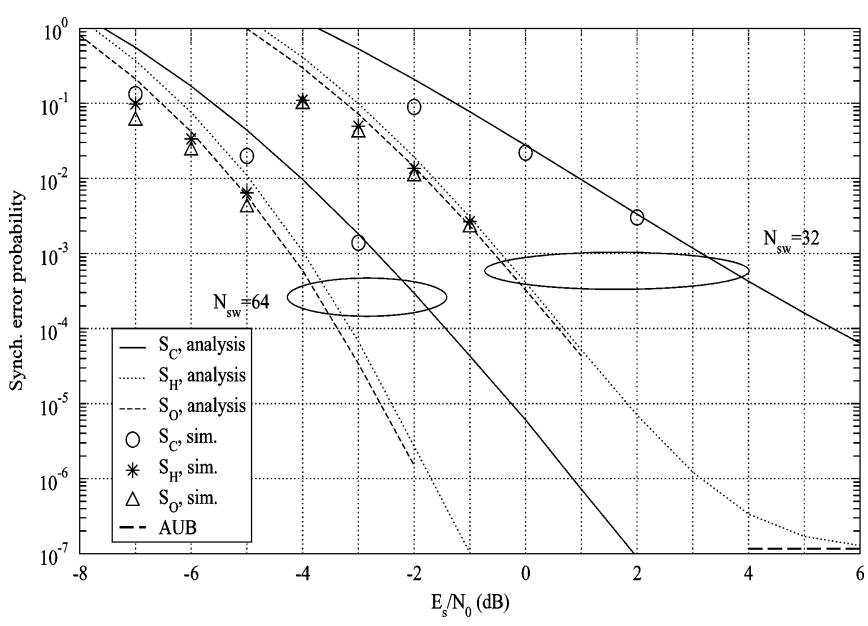

Fig. 4. False-synchronization probability versus $E_{s} / N_{0}=1 / 2 \sigma^{2}$. Sync word of length $N_{\mathrm{SW}}=32$ and $N_{\mathrm{SW}}=64$, frame length $N_{f}=1000$. The asymptotic union bounds are $\mathrm{AUB}=1.2 \times 10^{-7}$ for $N_{\mathrm{SW}}=32$ and $\mathrm{AUB}=$ $2.7 \times 10^{-17}$ for $N_{\mathrm{SW}}=64$ (not shown).

word with $N_{\mathrm{SW}}=24$ and frame length $N_{f}=240$ is considered in Fig. 3. In this figure, the bounds obtained with the metrics $S_{\mathrm{H}}$ and $S_{\mathrm{O}}$ are shown, together with the relevant simulation results. The performance evaluation of the correlation rule is reported for comparison, as well as the RDL bound and the asymptotic union bound. For simulation results, the sync word of length $N=24$ in [16] ( $050 C D$, in hexadecimal) has been considered.

Finally, Fig. 4 reports the synchronization error probability for $N_{\mathrm{SW}}=32, N_{\mathrm{SW}}=64$, and for frame length $N_{f}=1000$. The sync words considered for the simulation points shown to validate the analysis are the CCSDS [4] sequences $1 A C F F C 1 D$ and $034776 C 7272895 B 0$ (in hexadecimal). It can be noted that, as in the previous figure, the correlation metric performs very poorly with respect to $S_{\mathrm{O}}$ and $S_{\mathrm{H}}$. For example, for a sync word of length $N_{\mathrm{SW}}=32$ and a target false synchronization probability $\leq 10^{-3}$, frame synchronization using the correlation metric requires at least $E_{s} / N_{0} \simeq 3.2 \mathrm{~dB}$, whereas for the metrics $S_{\mathrm{O}}$ or $S_{\mathrm{H}}$ (these two perform very similarly) the minimum required $\mathrm{SNR}$ is $E_{s} / N_{0} \simeq-0.5 \mathrm{~dB}$, with a gain of around $3.7 \mathrm{~dB}$ in terms of SNR.

We note that our analytical bounds are tight to simulation results for the cases of interest and tend asymptotically to the exact values, allowing performance analysis of frame synchronization where simulation is not feasible.

\section{CONCLUSION}

Frame synchronization based on sync words, in the periodically embedded case, has been studied. Analytical bounds for the performance of optimum and suboptimum frame synchronization in AWGN channels have been provided. Curves of the PSEP and of the false synchronization probability are provided for sync word lengths of practical use. The numerical results show that the bounds, which represent a useful tool for the design of frame synchronizers, are very tight and tend asymptotically to the exact results.

\section{REFERENCES}

[1] R. H. Barker, "Group synchronization of binary digital systems," in Communication Theory. W. Jackson, Ed. London, U.K.: Butterworth, 1953.

[2] R. A. Scholtz, "Frame synchronization techniques," IEEE Trans. Commun., vol. COM-28, no. 8, pp. 1204-1213, Aug. 1980.

[3] C. Georghiades and E. Serpedin, Communication Handbook. Boca Raton, FL: CRC, 2002, ch. 18.

[4] "TM synchronization and channel coding," CCSDS Blue Book, no. 1, pp. 6-1-6-2, Sep. 2003.

[5] A. Ventura and T. Hindelang, "A low complexity algorithm for frame synchronization in TDD-LCR system," in Proc. IEEE PIMRC 2002, Lisbon, Portugal, Sep., pp. 1561-1565.

[6] J. L. Massey, "Optimum frame synchronization," IEEE Trans. Commun., vol. COM-20, no. 4, pp. 115-119, Apr. 1972.

[7] P. T. Nielsen, "Some optimum and suboptimum frame synchronizers for binary data in Gaussian noise," IEEE Trans. Commun., vol. COM-21, no. 6, pp. 770-772, Jun. 1973.

[8] G. L. Lui and H. H. Tan, "Frame synchronization for Gaussian channels," IEEE Trans. Commun., vol. COM-35, no. 8, pp. 818-829, Aug. 1987.

[9] B. H. Moon and S. S. Soliman, "ML frame synchronization for the Gaussian channel with ISI," in Proc. IEEE Int. Conf. Commun., ICC 1991, Paris, France, Jun., pp. 1698-1702.

[10] M. M. K. Howlader and B. D. Woerner, "Decoder-assisted frame synchronization for packet transmission," IEEE J. Sel. Areas Commun., vol. 19, no. 12 , pp. 2331-2345, Dec. 2001.

[11] A. Kopansky and M. Bystrom, "Frame synchronization for noncoherent demodulation on flat fading channels," in Proc. IEEE Int. Conf. Commun., ICC 2000, New Orleans, USA, Jun., pp. 312-316.

[12] P. F. Driessen, "Performance of frame synchronization in packet transmission using bit erasure information," IEEE Trans. Commun., vol. 39, no. 4, pp. 567-573, Apr. 1991.

[13] M. Chiani and M. G. Martini, "Practical frame synchronization for data with unknown distribution on AWGN channels," IEEE Commun. Lett., vol. 9 , no. 5, pp. 456-458, May 2005.

[14] M. Chiani and M. G. Martini, "On sequential frame synchronization in AWGN channels," IEEE Trans. Commun., vol. 54, no. 2, pp. 339-348, Feb. 2006.

[15] M. Chiani and M. G. Martini, "Analysis of optimum frame synchronization based on periodically embedded sync words," IEIIT, Univ. Bologna. Bologna, Italy, Tech. Rep. IEIIT-BO-06-06, Jul. 2006.

[16] J. L. Maury and F. Styles, "Development of optimum frame synchronization codes for Goddard space flight center PCM telemetry standards," in Proc. Nat. Telemetering Conf., Los Angeles, CA, Jun. 1964, pp. 1-10. 